\title{
Induction chemotherapy followed by concurrent chemoradiotherapy versus CCRT for locally advanced hypopharynx and base of tongue cancer
}

Sung Hee Lim ${ }^{1,2,}$, , Jong-Mu Sun ${ }^{2,}$, Joohyun Hong ${ }^{2}$, Dongryul Oh³ ${ }^{3}$ Yong Chan $\mathrm{Ahn}^{3}$, Man Ki Chung ${ }^{4}$, Han-Sin Jeong ${ }^{4}$, Young-Ik Son ${ }^{4}$, Myung-Ju Ahn², Chung-Hwan Baek ${ }^{4}$, and Keunchil Park ${ }^{2}$

\begin{abstract}
${ }^{1}$ Division of Hematology-Oncology, Department of Internal Medicine, Soonchunhyang University Bucheon Hospital, Bucheon; ${ }^{2}$ Division of Hematology-Oncology, Department of Medicine, Departments of ${ }^{3}$ Radiation Oncology and ${ }^{4}$ Otorhinolaryngology-Head and Neck Surgery, Samsung Medical Center, Sungkyunkwan University School of Medicine, Seoul, Korea
\end{abstract}

Received: May 15, 2019

Revised : September 4, 2019

Accepted: October 2, 2019

\section{Correspondence to}

Keunchil Park, M.D.

Division of Hematology-

Oncology, Department of

Medicine, Samsung Medical

Center, Sungkyunkwan

University School of Medicine,

81 Irwon-ro, Gangnam-gu, Seoul

06351, Korea

Tel.: +82-2-3410-3438

Fax: $+82-2-3410-1754$

E-mail:kpark@skku.edu

https://orcid.org/0000-0002-

4846-7449

*These authors contributed equally to this work.
Background/Aims: Clinical trials have not consistently supported the use of induction chemotherapy (IC) for locally advanced head and neck squamous cell cancer. Hypopharynx and base of tongue (BOT) cancer has shown relatively poor survival. We investigated the role of IC in improving outcome over current chemoradiotherapy (CRT) in patients with hypopharynx and BOT cancer.

Methods: Treatment-naïve patients with stage III/IV (Mo) hypopharynx or BOT cancer were randomly assigned to receive CRT alone (CRT arm: cisplatin $100 \mathrm{mg} / \mathrm{m}^{2}$ on D1 3-weekly, two times plus radiotherapy $68.4 \mathrm{~Gy} / 30$ fractions on weekdays) versus two 21-day cycles of IC with TPF (docetaxel \& cisplatin $75 \mathrm{mg} / \mathrm{m}^{2}$ on D1, and fluorouracil $75 \mathrm{mg} / \mathrm{m}^{2}$ on D1-4) followed by the same CRT regimen (IC arm). The primary endpoint was progression-free survival (PFS).

Results: This study closed early after enrollment of 36 patients (19 in the CRT arm, 17 in the IC arm). After a median follow-up of 47.2 months, there was no significant difference in PFS: the median PFS was 26.8 months for the CRT arm and was not reached for the IC arm $(p=0.13)$. However, the survival curves were widely separated with a plateau after 3 years, suggesting a potential survival benefit from IC: 3 -year PFS rates were $45 \%$ and $68 \%$, and 3-year overall survival rates were $56 \%$ and $86 \%$, in the CRT and IC arms, respectively.

Conclusions: This study failed to demonstrate that induction TPF chemotherapy improves survival in patients with BOT and hypopharynx cancer. However, it suggested a favorable outcome with IC to this population.

Keywords: Locally advanced; Hypopharyngeal neoplasms; Base of tongue cancer; Induction chemotherapy; Concurrent chemoradiotherapy

\section{INTRODUCTION}

A role for induction chemotherapy (IC) in locally advanced squamous cell head and neck cancer (LASCC) has not been fully demonstrated. Two recent randomized phase III trials did not prove a survival benefit of
IC compared to concurrent chemoradiotherapy (CRT) alone $[1,2]$, however the failure of those studies could be attributed to methodological problems such as a, relatively low recruitment rate. Still, $40 \%$ to $60 \%$ of patients with LASCC experience locoregional or distant metastases after CRT [3] and the 3-year survival rate was less than 
$50 \%$ in a meta-analysis establishing CRT as the current standard of care [4]. Various studies to evaluate the efficacy and tolerability of proper combinations and/or schedules of IC have been tried. Among them, docetaxel (T) combined with cisplatin, and 5-fluorouracil (PF) (TPF regimen) showed superior survival compared with the PF regimen (cisplatin + 5-fluorouracil) [5-7].

We hypothesized that some subgroup populations could benefit from integrating IC into CRT alone. Previously, we confirmed that long-term outcome was significantly worse in patients with hypopharyngeal cancer compared to those with oropharyngeal cancer (3-year overall survival [OS] rate 52\% vs. 75\%, $p=0.001$; 3 -year progression-free survival [PFS] rate $42 \%$ vs. $72 \%$, $p<0.001$ ), although the overall response rate (ORR) after CRT was fairly good in both groups [8]. And another phase III trial comparing concurrent CRT versus IC followed by CRT reported a more pronounced benefit of IC with a TPF regimen in patients with non-oropharyngeal cancer compared to overall LASCC patients [9].

In the present study, we investigated whether IC with two cycles of TPF regimen before definitive CRT improved PFS in locally advanced (Stage III \& IV [Mo]) hypopharyngeal and base of tongue (BOT) cancer compared to definitive CRT alone.

\section{METHODS}

\section{Patients and study design}

This phase II randomized trial (NCTo1312350) was conducted to test the efficacy of IC with a TPF regimen followed by concurrent chemoradiotherapy compared to CRT alone. Eligible patients were age $\geq 18$ years with histologically confirmed stage III or IV (Mo disease) hypopharyngeal or BOT cancer according to the American Joint Cancer Committee (AJCC) staging classification 7 th edition and had one or more measurable lesions. No previous chemotherapy, radiotherapy or surgery was allowed. Other key eligibility criteria included an Eastern Cooperative Oncology Group (ECOG) performance status of $\leq 2$ and adequate bone marrow, renal and hepatic function. Patients with distant metastasis were excluded. All patients provided informed consent. This study was approved by the Institutional Review Board of Samsung Medical Center (IRB no.: 2010-10-028).

\section{Study treatment}

Patients were randomly assigned to receive either IC followed by CRT (IC arm) or CRT only (CRT arm). IC consisted of two cycles of TPF regimen (docetaxel and cisplatin at $75 \mathrm{mg} / \mathrm{m}^{2}$ each on day 1 , and fluorouracil [5FU] at $750 \mathrm{mg} / \mathrm{m}^{2}$ on D1 to 4 , every 3 weeks), followed by CRT 3 to 5 weeks after the completion of IC. CRT was administered with cisplatin $100 \mathrm{mg} / \mathrm{m}^{2}$ on day 1 every 3 weeks up to a total of two to three times. Patients received radiotherapy using three-dimensional conformal radiotherapy or intensity-modulated radiotherapy techniques delivered at doses of 63 to 72 Gy in 30 to 35 fractions in 2.0 to 2.4 Gy per fraction over 6 to 7 weeks. For IC + CRT treated patients, neck computed tomography (CT) and positron emission tomography (PET)-CT were performed to assess the response to IC before starting CRT 3 weeks after completion of the TPF chemotherapy. If a patient had disease progression after IC, they were was referred to a multidisciplinary head and neck tumor board for further treatment. After completion of CRT in both the IC and CRT arm, a neck CT was performed after 1 month and then every 3 months for 1 year and every 6 months thereafter. PET-CT was performed after 4 months and repeated every 6 months for 2 years and every 2 years thereafter until disease progression or death. If patients showed disease progression during or after study treatment, further treatment could be administered at the discretion of the investigators.

\section{Endpoints}

The primary endpoint was PFS, defined as the time from randomization to progression, secondary cancer or death resulting from any cause. Secondary endpoints included ORRs, OS, and toxicity. OS was calculated from the time of randomization to the date of death. PFS was set to be primary endpoint due to a surrogate marker of OS. These endpoints were measured in all registered patients (i.e., the intention-to-treat population). Response Evaluation Criteria in Solid Tumors (RECIST version 1.1) and adverse events (AEs) were assessed according to National Cancer Institute criteria (CTCAE v3).

\section{Statistical analysis}

Survival rates were estimated by the Kaplan-Meier method and compared between the two treatment groups using the log-rank test. The sample size for the trial was 
derived from following assumption. Given a one-tailed alpha of 0.1 and power of 0.85 , the 1-year PFS rate would be $65 \%$ and $80 \%$ in the CRT arm and IC arm, respectively, with an accrual rate of 29 patients per year and an additional follow-up period of 1.5 years. A 10\% ineligible or non-assessable rate was assumed, resulting in the accrual goal of 100 total patients (50 patients for each arm). The significance of statistical tests was evaluated based on a one-tailed alpha value of $10 \%$ for the primary endpoint and a two-tailed alpha value of $5 \%$ for the other endpoints. Statistical analyses were performed using SPSS version 21 (IBM Co., Armonk, NY, USA). This trial was closed early due to slow recruitment.

\section{RESULTS}

\section{Study patients}

Between December 2010 and April 2015, a total of 41 patients were screened. Five patients were excluded during the screening period. We randomly assigned 36 patients to receive IC followed by CRT or CRT only (Fig. 1). Patient baseline characteristics are shown in Table 1 and were well balanced between the two groups. About twothirds of all patients were diagnosed with hypopharyngeal cancer and the rest were BOT cancers. All had squamous cell carcinoma histology, 28 patients $(78 \%)$ had N2 to $\mathrm{N}_{3}$ disease and approximately $80 \%$ of patients showed clinical stage IV (A/B) disease (AJCC 7th edition).

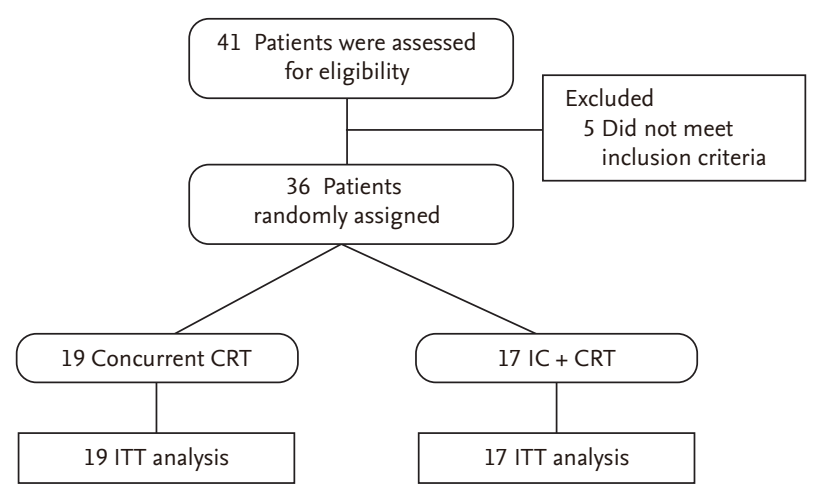

Figure 1. Flow chart of study schema. CRT, chemoradiotherapy; IC, induction chemotherapy; ITT, intention-to-treat.

\section{Efficacy}

At the time of data analysis (February 2017), a total of 15 patients (42\%) had disease progression or recurrent disease after partial response or complete response to treatment. After a median follow-up of 47.2 months, 1-year estimated PFS rates were 63.2\% (95\% confidence interval [CI], 0.415 to 0.848 ) in the CRT group and $76.5 \%$ (95\% CI, 0.563 to 0.966 ) in the IC group, respectively. The median PFS was 26.8 months in the CRT arm and not reached in the IC arm ( $p=0.131$ ) (Fig. 2A). Two-year OS rates were $72.2 \%$ (95\% CI, 0.515 to 0.929 ) and $86.9 \%$ (95\% CI, 0.698 to 1.040) in the CRT and IC arms, respectively. Three-years after randomization, the survival curves widely separated with a plateau: 3-year PFS estimates were $45 \%$ and $68 \%$ (hazard ratio [HR], 0.55; 95\% CI, 0.19 to 1.60 ), and 3-year OS estimates were $56 \%$ and $86 \%$ (HR, 0.35 ; $95 \%$ CI, 0.07 to 1.69), in the CRT and IC arms, respectively (Fig. 2B).

In 23 patients with hypopharynx cancer, IC tended to show more improvement in PFS compared to BOT cancer, although neither were statistically significant ( $p=0.198$ and $p=0.217$ ) (Fig. 3). There was no significant difference in the overall incidence of distant failure between the two groups $(p=0.324)$. Two patients in the CRT arm and three in the IC arm initially progressed at distant metastatic sites.

The ORR to IC was 94.1\%; one patient (5.8\%) achieved complete response, $88.2 \%$ had partial responses and one patient (5.8\%) had stable disease. None of the patients progressed on IC. After completion of CRT, among all 36 patients, 20 reached complete response (55.6\%), 14 had partial responses (38.9\%) and one had stable disease (2.8\%). Overall response rates were $100 \%$ in the CRT group and $88.2 \%$ in the IC group $(p=0.216)$. Five patients underwent salvage operations (four CRT arm, one IC arm) and four patients received salvage chemotherapy after progression.

\section{Toxicity}

All patients were monitored for AEs and 94.4\% (34/36) of them experienced an $\mathrm{AE}$ during the study period. There was only one case of grade $\geq 3 \mathrm{AE}$ of stomatitis (5.9\%) in the IC group during the CRT phase. The most common AEs experienced in both treatment arms were anemia, stomatitis, dry mouth, and dysphagia (Table 2). The addition of IC before CRT was relatively well tol- 
Table 1. Baseline patient characteristics

\begin{tabular}{|c|c|c|}
\hline Characteristic & $\begin{array}{l}\text { CRT arm } \\
(\mathrm{n}=19)\end{array}$ & $\begin{array}{c}\mathrm{IC} \rightarrow \text { CRT arm } \\
(\mathrm{n}=17)\end{array}$ \\
\hline \multicolumn{3}{|l|}{ Age, yr } \\
\hline Mean \pm SD & $61.7 \pm 9.54$ & $60.0 \pm 10.05$ \\
\hline Range & $42-72$ & $43-76$ \\
\hline \multicolumn{3}{|l|}{ Sex, n (\%) } \\
\hline Male & $15(78.9)$ & $15(88.2)$ \\
\hline Female & $4(21.1)$ & $2(11.8)$ \\
\hline \multicolumn{3}{|c|}{$\begin{array}{l}\text { ECOG performance } \\
\text { status, } \mathrm{n}(\%)\end{array}$} \\
\hline o & $2(10.5)$ & $1(5 \cdot 9)$ \\
\hline 1 & $17(89 \cdot 5)$ & $16(94.1)$ \\
\hline \multicolumn{3}{|l|}{ Histology, n (\%) } \\
\hline $\begin{array}{l}\text { Squamous cell } \\
\text { carcinoma }\end{array}$ & $19(100.0)$ & $17(100.0)$ \\
\hline \multicolumn{3}{|c|}{ Differentiation, n (\%) } \\
\hline Moderate & $6(31.6)$ & $3(17.6)$ \\
\hline Poorly & $2(10.5)$ & $2(11.8)$ \\
\hline Unknown & $11(57 \cdot 9)$ & $12(70.6)$ \\
\hline \multicolumn{3}{|l|}{ Tumor stage, n (\%) } \\
\hline $\mathrm{Tl}$ & $2(10.5)$ & $\mathrm{O}$ \\
\hline $\mathrm{T} 2$ & $7(36.8)$ & $7(41.2)$ \\
\hline $\mathrm{T}_{3}$ & $6(31.6)$ & $6(35 \cdot 3)$ \\
\hline $\mathrm{T}_{4}$ & $4(21.1)$ & $4(23.5)$ \\
\hline \multicolumn{3}{|l|}{ Nodal stage, n(\%) } \\
\hline No & $2(10.5)$ & $1(5 \cdot 9)$ \\
\hline $\mathrm{N} 1$ & $2(10.5)$ & $3(17 \cdot 6)$ \\
\hline $\mathrm{N} 2$ & $15(78.9)$ & $12(70.6)$ \\
\hline $\mathrm{N}_{3}$ & o & $1(5 \cdot 9)$ \\
\hline \multicolumn{3}{|l|}{ AJCC stage, n (\%) } \\
\hline III & $4(21.1)$ & $4(23.5)$ \\
\hline IV & $15(78.9)$ & $13(76.5)$ \\
\hline IVA/IVB & $15 / 0$ & $12 / 1$ \\
\hline \multicolumn{3}{|c|}{ Primary tumor site, $\mathrm{n}(\%)$} \\
\hline Hypopharynx & $12(63.2)$ & $11(64.7)$ \\
\hline Base of tongue & $7(36.8)$ & $6(35 \cdot 3)$ \\
\hline
\end{tabular}

CRT, chemoradiotherapy; IC, induction chemotherapy; ECOG, Eastern Cooperative Oncology Group; AJCC, American Joint Cancer Committee.

erated and all AEs were manageable. Dose reductions of chemotherapy were required in $29.4 \%$ during IC and $5.9 \%$ during concurrent chemoradiotherapy only in the
IC arm. Dose delays of at least one cycle of chemotherapy happened in $15.8 \%$ and $29.4 \%$ of patients during the concurrent chemoradiotherapy period in the CRT and IC arms, respectively (Table 3).

\section{Treatment and drug delivery}

The total number of cisplatin chemotherapy cycles administered was 47 (mean, 2.5) in the CRT arm and 42 (mean, 2.5) in the IC arm. Two cycles of planned IC were performed in all patients on the IC arm. The delivered relative dose intensities of radiation and cisplatin for CRT were similarly high in both groups (98.9\%, 98.8\% in the CRT am and 93.5\%, $92.8 \%$ in the IC arm, respectively) (Table 3). The mean cumulative dose of cisplatin during CRT was $242 \mathrm{mg} / \mathrm{m}^{2}$ in the CRT arm and $213 \mathrm{mg} /$ $\mathrm{m}^{2}$ in the IC arm $(p=0.096)$. Three chemotherapeutic agents were given to IC group patients with quite high dose intensities ( $\geq 95 \%$ ).

\section{DISCUSSION}

We initially designed this phase II trial to evaluate the efficacy of adding IC to concurrent CRT in a specific patient subset of BOT or hypopharynx cancer. While the TAX (Cisplatin and Fluorouracil Alone or with Docetaxel in Head and Neck Cancer) 324 trial suggested that TPF IC was superior to a PF regimen with improved survival, we noticed that the 3-year OS rate in the TAX 324 trial, which included about 50\% oropharyngeal cancer patients, was $62 \%$ in the TPF IC group [6], but only $49 \%$ in a subgroup analysis of hypopharynx cancer patients [10]. Such an inferior outcome for hypopharyngeal cancer was similar to our previous study [8] where the 3-year OS rate was $52 \%$ in the hypopharynx group compared to $75 \%$ in the oropharynx group (HR, 2.21; $95 \%$ CI, 1.38 to 3.56; $p=0.001$ ) after treatment with upfront CRT.

Hypopharynx and BOT cancers have been regarded as good candidates for organ preservation, and so multidisciplinary treatments including chemotherapy, and radiotherapy rather than surgery have been evaluated. IC before CRT demonstrates a superior organ preservation rate in hypopharynx and BOT cancer (3-year PFS: 52\%) [11] and the TPF regimen showed a better ORR and a 3-year larynx preservation rate of 70.3\% compared to the $\mathrm{PF}$ regimen [12,13]. 

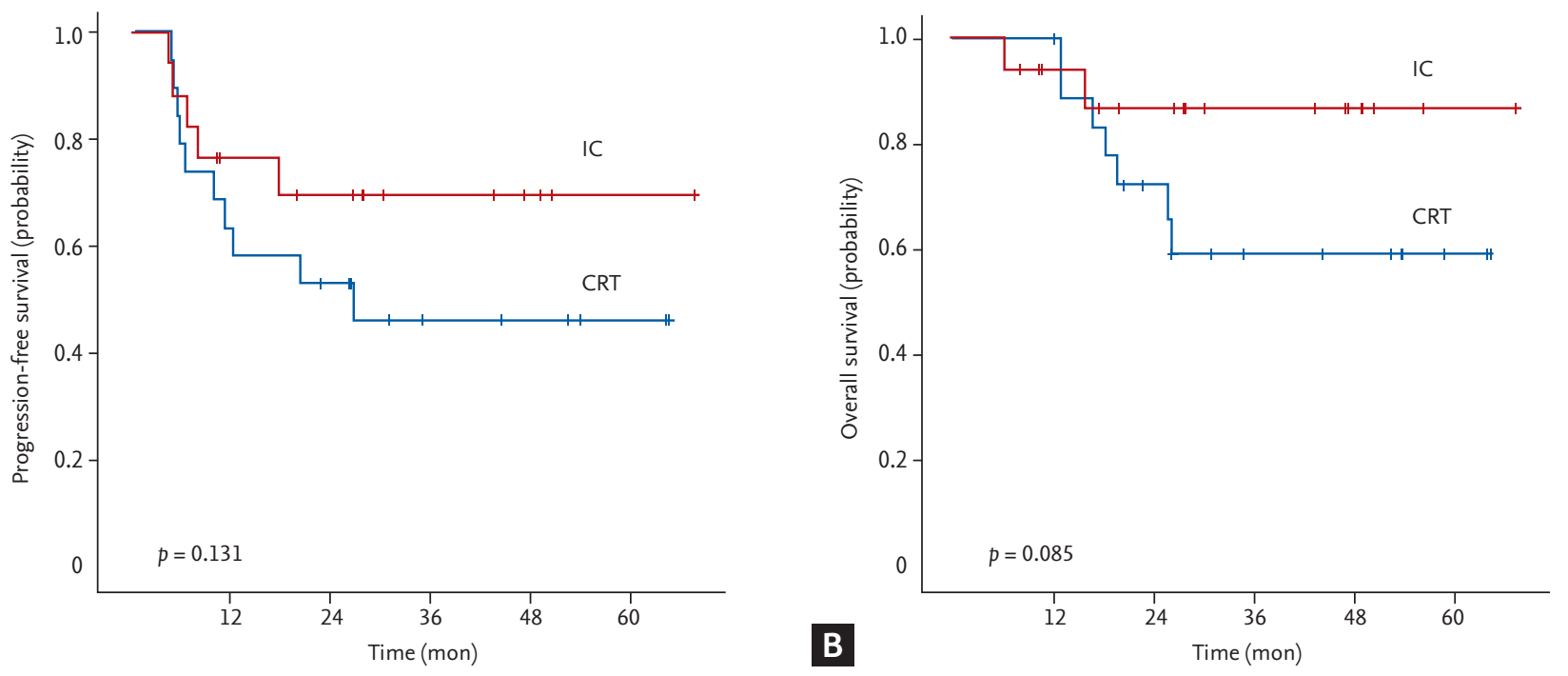

Figure 2. Kaplan-Meier survival curves by treatment arm and corresponding p values. (A) Progression-free survival, (B) overall survival. IC, induction chemotherapy; CRT, chemoradiotherapy.

\section{A}

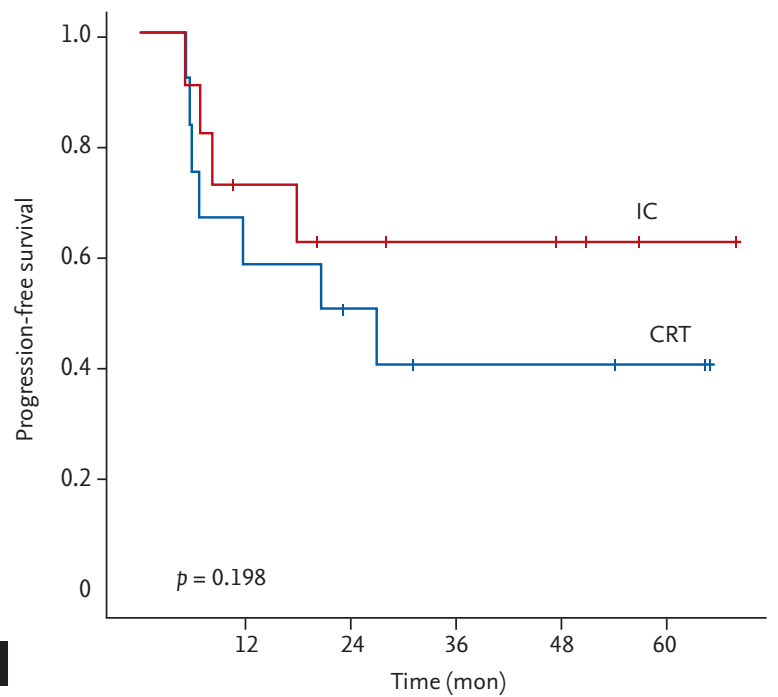

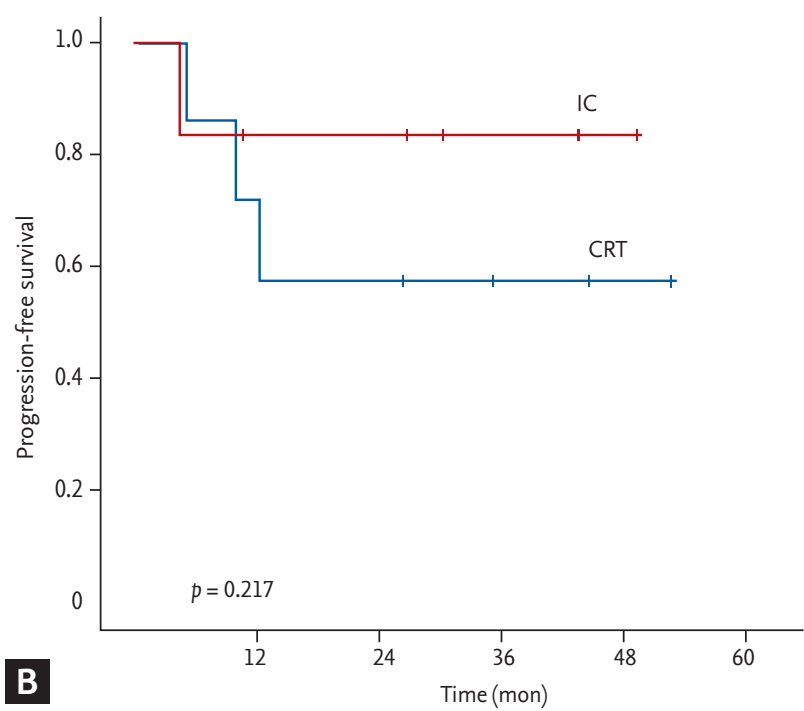

Figure 3. Subset analysis of progression-free survival for patients with (A) hypopharyngeal cancer, (B) base of tongue cancer. IC, induction chemotherapy; CRT, chemoradiotherapy.

We administered two cycles of IC with the TPF regimen as previously reported [7]. In both treatment arms, concurrent chemoradiotherapy was delivered with a 3-weekly cisplatin dose of $100 \mathrm{mg} / \mathrm{m}^{2}$, a current standard treatment. Importantly, the study treatment was tolerated well without any $\mathrm{Gr}_{4}$ toxicities even after the completion of chemoradiotherapy. In the present study, which was prematurely closed due to slow accrual with a small number of patients, the primary endpoint of $15 \%$ improvement in 1-year PFS was not met. However, 1-year PFS rates were $63.2 \%$ (95\% CI, 0.415 to 0.848) in the CRT group and $76.5 \%$ (95\% CI, 0.563 to 0.966 ) in the IC group, which supports the trend favoring IC. However, we could not validate the result due to early closure of the study. While the present study was ongoing, results from related randomized phase III trials (PARADIGM 
Table 2. Toxicity profile

\begin{tabular}{|c|c|c|c|c|}
\hline \multirow{3}{*}{ Toxicity } & \multicolumn{4}{|c|}{ NCI-CTC grade } \\
\hline & \multicolumn{2}{|c|}{ CRT $\operatorname{arm}(\mathrm{n}=19)$} & \multicolumn{2}{|c|}{$\mathrm{IC}+\mathrm{CRT} \operatorname{arm}(\mathrm{n}=17)$} \\
\hline & $\mathrm{Gl}-2$ & $\mathrm{G}_{3}-4$ & $\mathrm{G} 1-2$ & $\mathrm{G}_{3}-4$ \\
\hline \multicolumn{5}{|l|}{ Hematologic, n (\%) } \\
\hline Neutropenia & - & - & - & - \\
\hline Febrile neutropenia & - & - & - & - \\
\hline Anemia & $18(94.7)$ & - & $16(94.1)$ & - \\
\hline Leukopenia & - & - & - & - \\
\hline Thrombocytopenia & - & - & - & - \\
\hline \multicolumn{5}{|l|}{ Non-hematologic, n (\%) } \\
\hline Nausea & $2(10.5)$ & - & $5(29.4)$ & - \\
\hline Vomiting & - & - & $4(23 \cdot 5)$ & - \\
\hline Diarrhea & $1(5 \cdot 3)$ & - & $4(23.5)$ & - \\
\hline Constipation & $2(10.5)$ & - & $4(23 \cdot 5)$ & - \\
\hline Stomatitis & $13(68.4)$ & - & $13(76.5)$ & $1(5.9)^{\mathrm{a}}$ \\
\hline Mucositis & - & - & - & - \\
\hline Peripheral neuropathy & - & - & - & - \\
\hline Alopecia & $1(5 \cdot 3)$ & - & $5(29.4)$ & - \\
\hline Bleeding & $1(5 \cdot 3)$ & - & - & - \\
\hline Dyspnea & - & - & $1(5.9)$ & - \\
\hline Fatigue & $1(5 \cdot 3)$ & - & - & - \\
\hline Anorexia & - & - & $1(5.9)$ & - \\
\hline Dry mouth & $11(57 \cdot 9)$ & - & $14(82.4)$ & - \\
\hline Skin rash & $1(5 \cdot 3)$ & - & $4(23 \cdot 5)$ & - \\
\hline Dysphagia & $14(73.7)$ & - & $16(94.1)$ & - \\
\hline
\end{tabular}

NCI-CTC, National Cancer Institute-Common Toxicity Criteria; CRT, chemoradiotherapy; IC, induction chemotherapy. ${ }^{\mathrm{a}}$ It was developed during CRT phase.

and DeCIDE) did not demonstrate the superiority of IC over CRT. There has also been a trend in favor of chemoradiotherapy alone. Accordingly, co-investigators hesitated to participate in our trial. In addition, among all head and neck squamous cell carcinoma, hypopharynx and BOT cancers are diagnosed infrequently and incidence rates have recently been decreasing [14].

Given that more than $50 \%$ of enrolled patients had oropharynx cancer in both the PARADIGM and DeCIDE study and the outcome of non-significant trends for improved survival in the non-oropharynx subgroup in the DeCIDE analysis [1,2], we hypothesized that IC might be useful for a selective subset of LASCC patients with poor prognosis.

Although PFS and OS in the two treatment arms of the present study were not significantly different, there was a trend for improved survival with TPF IC (HR, 0.35; 95\% $\mathrm{CI}, 0.07$ to 1.69$)$, reaching a plateau after 3 years. The OS rate of $86 \%$ at 3 years in the IC group compares favorably to other reported data $[1,2]$, particularly given that $78 \%$ of cases ( 28 of 36$)$ had stage IVA/B disease in the current study.

The relatively small number of patients and early termination of this study limits interpretation, but patients in both groups showed good adherence during the treatment phase, which led to a higher relative mean dose intensity of chemotherapy and radiotherapy. Among about $35 \%$ of patients with BOT cancer in both group, human papillomavirus (HPV) status were not evaluated which has been regarded as an important 
Table 3. Treatment delivery

\begin{tabular}{|c|c|c|c|}
\hline \multirow{2}{*}{ Drug/radiotherapy delivery } & \multirow{2}{*}{$\begin{array}{l}\text { CRT arm }(\mathrm{n}=19) \\
\text { Concurrent CRT }\end{array}$} & \multicolumn{2}{|c|}{$\mathrm{IC} \operatorname{arm}(\mathrm{n}=17)$} \\
\hline & & Induction chemotherapy & Concurrent CRT \\
\hline \multicolumn{4}{|l|}{ Chemotherapy cycles } \\
\hline Number & 47 & 34 & 42 \\
\hline Mean & 2.5 & 2 & 2.5 \\
\hline \multicolumn{4}{|l|}{ Radiotherapy } \\
\hline Mean \pm SD, cGy & $6,799 \pm 208.5$ & NA & $6,448 \pm 1,298.4$ \\
\hline Dose intensity per patient, mean, \% & 98.9 & NA & $93 \cdot 5$ \\
\hline \multicolumn{4}{|l|}{ Relative dose-intensity per patient } \\
\hline Cisplatin, mean, \% & 98.8 & NA & 92.8 \\
\hline Docetaxel, mean, \% & NA & 96.2 & NA \\
\hline Cisplatin, mean, \% & NA & 96.2 & NA \\
\hline Fluorouracil, mean, \% & NA & $97 \cdot 3$ & NA \\
\hline Dose reductions in at least one cycle, $\mathrm{n}(\%)$ & - & $5(29.4)$ & $1(5 \cdot 9)$ \\
\hline Delays in at least one cycle, $\mathrm{n}(\%)$ & $3(15.8)$ & - & $5(29.4)$ \\
\hline
\end{tabular}

CRT, chemoradiotherapy; IC, induction chemotherapy; SD, standard deviation; NA, not applicable.

prognostic factor in HNSCC.

In summary, our study failed to demonstrate a significant improvement of PFS by adding IC before chemoradiotherapy, and concurrent CRT remains the standard of care with IC remaining investigational. Considering the PFS trend favoring IC, further prospective randomized clinical trials in a large number of patients are warranted in specific subset of LASCC patients to improve poor outcomes.

\section{KEY MESSAGE}

1. Hypopharynx and base of tongue cancer (BOT) shows relatively poor survival.

2. Induction docetaxel, cisplatin and fluorouracil chemotherapy did not prolong progression-free survival compared to concurrent chemoradiotherapy alone among hypopharynx and BOT cancer patients; however, after 3-year, favorable outcome with induction chemotherapy was sustained.

\section{Conflict of interest}

Keunchil Park has received honoraria from Abbvie, Astellas, Astra Zeneca, Boehringer Ingelheim, Clovis, Eli
Lilly, GSK, Hanmi, Kyowa Hakko Kirin, MSD, ONO, and Roche. Keunchil Park's institution received research funding from Astra Zeneca.

\section{REFERENCES}

1. Cohen EE, Karrison TG, Kocherginsky M, et al. Phase III randomized trial of induction chemotherapy in patients with $\mathrm{N}_{2}$ or $\mathrm{N}_{3}$ locally advanced head and neck cancer. J Clin Oncol 2014;32:2735-2743.

2. Haddad R, O'Neill A, Rabinowits G, et al. Induction chemotherapy followed by concurrent chemoradiotherapy (sequential chemoradiotherapy) versus concurrent chemoradiotherapy alone in locally advanced head and neck cancer (PARADIGM): a randomised phase 3 trial. Lancet Oncol 2013;14:257-264.

3. Denis F, Garaud P, Bardet E, et al. Final results of the 94or French Head and Neck Oncology and Radiotherapy Group randomized trial comparing radiotherapy alone with concomitant radiochemotherapy in advanced-stage oropharynx carcinoma. J Clin Oncol 2004;22:69-76.

4. Pignon JP, le Maitre A, Maillard E, Bourhis J; MACHNC Collaborative Group. Meta-analysis of chemotherapy in head and neck cancer (MACH-NC): an update on 93 randomised trials and 17,346 patients. Radiother Oncol 
2009;92:4-14.

5. Hitt R, Lopez-Pousa A, Martinez-Trufero J, et al. Phase III study comparing cisplatin plus fluorouracil to paclitaxel, cisplatin, and fluorouracil induction chemotherapy followed by chemoradiotherapy in locally advanced head and neck cancer. J Clin Oncol 2005;23:8636-8645.

6. Posner MR, Hershock DM, Blajman CR, et al. Cisplatin and fluorouracil alone or with docetaxel in head and neck cancer. N Engl J Med 2007;357:1705-1715.

7. Vermorken JB, Remenar E, van Herpen C, et al. Cisplatin, fluorouracil, and docetaxel in unresectable head and neck cancer. N Engl J Med 2007;357:1695-1704.

8. Lim SH, Lee SJ, Ahn MJ, Park K, Sun JM. Different clinical outcomes between locally advanced hypopharyngeal and oropharyngeal cancer treated with definitive concurrent chemoradiotherapy: implication for subgroup selection for induction chemotherapy. Jpn J Clin Oncol 2016;46:40-45.

9. Ghi MG, Paccagnella A, Ferrari D, et al. Concomitant chemoradiation (CRT) or cetuximab/RT (CET/RT) versus induction docetaxel/cisplatin/5-fluorouracil (TPF) followed by CRT or CET/RT in patients with Locally
Advanced Squamous Cell Carcinoma of Head and Neck (LASCCHN). A randomized phase III factorial study (NCTo1086826). J Clin Onclol 2014;32(15 Suppl):6004.

10. Posner MR, Norris CM, Wirth LJ, et al. Sequential therapy for the locally advanced larynx and hypopharynx cancer subgroup in TAX 324: survival, surgery, and organ preservation. Ann Oncol 2009;20:921-927.

11. Urba SG, Moon J, Giri PG, et al. Organ preservation for advanced resectable cancer of the base of tongue and hypopharynx: a Southwest Oncology Group trial. J Clin Oncol 2005;23:88-95.

12. Pointreau Y, Garaud P, Chapet S, et al. Randomized trial of induction chemotherapy with cisplatin and 5-fluorouracil with or without docetaxel for larynx preservation. J Natl Cancer Inst 2009;101:498-506.

13. Janoray G, Pointreau Y, Garaud P, et al. Long-term results of a multicenter randomized phase III trial of induction chemotherapy with cisplatin, 5-fluorouracil, \pm docetaxel for larynx preservation. J Natl Cancer Inst 2015;108:djv368.

14. Mifsud M, Eskander A, Irish J, et al. Evolving trends in head and neck cancer epidemiology: Ontario, Canada 1993-2010. Head Neck 2017;39:1770-1778. 